\title{
Response to perturbations for granular flow in a hopper
}

\author{
John F. Wambaugh* and Robert P. Behringer \\ Department of Physics and Center for Nonlinear and Complex Systems, Duke University, Durham, North Carolina 27708, USA
}

John V. Matthews

Department of Mathematics, University of Tennessee at Chattanooga, Chattanooga, Tennessee 37403, USA

Pierre A. Gremaud

Department of Mathematics and Center for Research in Scientific Computation, North Carolina State University, Raleigh, North Carolina 27695-8205, USA

(Received 12 January 2007; revised manuscript received 13 July 2007; published 14 November 2007)

\begin{abstract}
We experimentally investigate the response to perturbations of circular symmetry for dense granular flow inside a three-dimensional right-conical hopper. These experiments consist of particle tracking velocimetry for the flow at the outer boundary of the hopper. We are able to test commonly used constitutive relations and observe granular flow phenomena that we can model numerically. Unperturbed conical hopper flow has been described as a radial velocity field with no azimuthal component. Guided by numerical models based upon continuum descriptions, we find experimental evidence for secondary, azimuthal circulation in response to perturbation of the symmetry with respect to gravity by tilting. For small perturbations we can discriminate between constitutive relations, based upon the agreement between the numerical predictions they produce and our experimental results. We find that the secondary circulation can be suppressed as wall friction is varied, also in agreement with numerical predictions. For large tilt angles we observe the abrupt onset of circulation for parameters where circulation was previously suppressed. Finally, we observe that for large tilt angles the fluctuations in velocity grow, independent of the onset of circulation.
\end{abstract}

DOI: 10.1103/PhysRevE.76.051303

PACS number(s): 45.70.-n, 47.57.Gc, 83.80.Fg, 47.85.M-

\section{INTRODUCTION}

Many practical processes involve the flow of dense granular materials. Applications range from food grains, ores, and coal to pharmaceutical powders. To predict flows of dense granular states, one would like a homogenized-continuum model that reflects the complexity of grain-scale interactions but can be applied at much larger scales. Continuum approaches frequently describe granular materials using elastoplastic models in which the material can support shear stresses up to a certain value before yielding and deforming irreversibly. In the simplest case, this approach amounts to Coulomb's law of friction.

The most common yield criterion is the Mohr-Coulomb criterion, which proposes a linear relationship between the magnitudes of normal stress $\sigma$ and shear stress $\tau$ in two dimensions. Because the choice of axes dictates the relative magnitude of the normal and shear stresses, the range of possible axes maps out a circle in the space of normal and shear stress- the "Mohr circle." The diameter of this circle is proportional to the magnitude of the stresses [1].

In the normal-shear stress space, the Mohr-Coulomb yield criterion is the straight line $\tau=\mu \sigma+c$, where $\mu$ is the coefficient of friction and $c$ is the cohesion. It follows for cohesionless granular materials, the case we consider here, that the yield criterion must pass through the origin. The stress

\footnotetext{
*Present address: National Center for Computational Toxicology, US EPA, Research Triangle Park, NC 27711; wambaugh@phy.duke.edu
}

within the material, and hence the diameter of the Mohr circle, can increase only until the yield criterion is tangent to the circle. The point of contact then indicates an axis of a coordinate frame in which the ratio of shear to normal stress (equivalent to the mobilization of friction) is maximized. In this two-dimensional analysis of a three-dimensional material, the direction of the maximally mobilized shear stress and the third, neglected, axis span the "mobilized plane" along which motion of the granular material is most likely in response to stress [1].

There are several approaches that have taken for generalizing the Mohr circle picture to three dimensions. An example is the Matsuoka-Nakai criterion. By geometrically averaging the three planes obtained by considering pairs of dimensions a "spatially mobilized plane" is obtained. This is similar to the generalization of the Tresca failure criterion for metals to three dimensions by von Mises [2]. Triaxial experiments support the validity of the Matsuoka-Nakai criterion, indicating that it may capture the average behavior of a granular material [3].

Unfortunately, the mathematics of continuum descriptions of granular materials are rife with instabilities, ill-posedness, and complex nonlinearities [4]. This means that evaluating the response of granular materials to perturbations that cause unusual behavior in continuum models is necessary to determine the physical relevance of such approaches.

The right-conical hopper provides an ideal test system for granular flow in that it is not only an experimentallyaccessible system, but also because the behavior is commonly assumed to tend to a steady state with known velocity and stress field solutions derived from soil mechanics. The so-called Jenike radial solutions describe the velocity and 
stress fields in an infinite hopper as self-similar functions of radial position alone, without nonradial velocity components [5]. In two dimensions, such soil mechanics descriptions of wedge hoppers have been studied extensively both experimentally [6-9] and theoretically $[10,11]$. Three-dimensional, right-conical hopper flow has been studied through experiments and discrete element simulations in the past $[12,13]$, but these studies did not make direct comparison to soil mechanics predictions.

Recent numerical work has taken advantage of the selfsimilar nature of the solution even in relatively general threedimensional geometries such as "pyramidal" hoppers. By perturbing the geometry of the simulated hopper, the response of the continuum description can be examined. In particular, numerical work has found that secondary, nonradial circulation currents arise. These currents depend sharply upon model parameters in nontrivial ways [14-16].

We make quantitative experimental observations of a right-conical granular hopper to determine if these numerically predicted behaviors, the result of nonlinearities in the continuum description of granular materials, have physical significance. We investigate both the influence of tilt-induced perturbations and changes in boundary roughness. By comparing with numerical predictions we investigate the ability of different constitutive relations to correctly model experimentally observed phenomena. Since we obtain localized time-resolved velocities, we also identify useful statistical characterizations of flowing dense granular matter.

\section{SOIL MECHANICS APPROACHES TO GRANULAR MATTER}

Before turning to the experiments, we give a brief summary of the relevant soil mechanics constitutive relations. At issue are the determining equations for the stress tensor. Force balance gives six equations for the nine components of the three-dimensional stress tensor. To close this system of equations we need additional constitutive relations describing the yielding, plasticity, and flow of the material [15]. Consideration of the velocity field $v$ describing the flow introduces three more unknowns bringing the necessary number of additional constraint equations to six.

We use Levy's flow rule to introduce five constraints $[1,17]$. Missing is a final constitutive relation describing the stress sufficient to cause plastic rearrangement, or yield, within the material [18].

In metal plasticity, the von Mises failure criterion is commonly used as a constitutive relation. The von Mises yield surface in stress space, however, is independent of the total pressure:

$$
\left(\sigma_{1}-\sigma_{2}\right)^{2}+\left(\sigma_{2}-\sigma_{3}\right)^{2}+\left(\sigma_{3}-\sigma_{1}\right)^{2}=c^{2},
$$

where $\sigma_{1}, \sigma_{2}$, and $\sigma_{3}$ are the principal stresses denoted in order of magnitude and $c$ is a constant. This is necessarily at odds with the Coulomb nature of granular failure. Replacing the constant term on the right-hand side by a term involving the principal stresses generalizes the von Mises criterion to granular materials by the introduction of a pressuredependent yield surface:

$$
\left(\sigma_{1}-\sigma_{2}\right)^{2}+\left(\sigma_{2}-\sigma_{3}\right)^{2}+\left(\sigma_{3}-\sigma_{1}\right)^{2}=6 \sin \theta_{s}^{2} I_{1}^{2}
$$

or, equivalently,

$$
9 I_{1}^{2}+2 I_{2}=6 \sin \theta_{s}^{2} I_{1}^{2},
$$

where the three stress invariants are $I_{1}=\frac{1}{3}\left(\sigma_{1}+\sigma_{2}+\sigma_{3}\right)$ (equivalent to the isotropic pressure), $I_{2}=-\left(\sigma_{2} \sigma_{3}+\sigma_{3} \sigma_{1}\right.$ $\left.+\sigma_{1} \sigma_{2}\right)$, and $I_{3}=\sigma_{1} \sigma_{2} \sigma_{3}$ and $\theta_{s}$ is the internal angle of friction of the sand $[1,2]$. This "granular von Mises" condition is commonly used as the missing granular constitutive relation.

As an alternative, Matsuoka and Nakai [2] apply the Mohr-Coulomb failure criterion $\left|\sigma_{j k}\right| \leq \tan \phi_{i} \sigma_{k k}$ for a cohesionless material to obtain, for each pair of axes $j$ and $k$, a Mohr circle with radii $R_{i}=\left(\sigma_{j}-\sigma_{k}\right) \sin \theta_{s}$, and a yield criterion that makes an angle $\phi_{i}$ at the origin such that $\cos \phi_{i}$ $=\left|\sigma_{j}-\sigma_{k}\right| / R_{i}$. They argue for the following constitutive relation:

$$
\tan \phi_{1}^{2}+\tan \phi_{2}^{2}+\tan \phi_{3}^{2}=c,
$$

which they show to be equivalent to

$$
I_{1} I_{2} / I_{3}=c .
$$

Gremaud et al. have computed flows in relatively general geometries for both of the above plasticity models $[15,16]$. The authors model granular flow in a vertically oriented infinite right-conical hopper described in spherical coordinates where $r$ is the distance from the origin, which is at the tip of the cone, $\theta$ is the angle measured away from the axis of symmetry, and $\phi$ is the azimuthal position about the symmetry axis. The authors vary the wall angle azimuthally about the constant angle $\theta_{w}$ as $\theta=\theta_{w}+\epsilon \cos m \phi$. The case $m=1$ corresponds to elongating the cross section of the hopper in a manner that is roughly similar to the effect of tilting the hopper with respect to gravity. Assuming $\epsilon$ is small (between $-5^{\circ}$ and $5^{\circ}$ for a tilted hopper), the velocity can be expanded as $v=v^{0}+\epsilon v^{1}+\cdots$ giving the radial and azimuthal components of the velocity as

$$
\begin{gathered}
v_{r} \approx v_{r}^{0}+\epsilon v_{r}^{1}, \\
v_{\phi} \approx \epsilon v_{\phi}^{1},
\end{gathered}
$$

where an unperturbed $(\epsilon=0)$ hopper has Jenike radial flow.

Two main observations were drawn from the numerical experiments of Gremaud et al. First, when vertical axisymmetry is broken, secondary circulation takes place, i.e., $v_{\phi}$ becomes nonzero and leads to two counter-rotating cells perpendicular to the tilt axis, as in Fig. 1. Second, the computed circulation currents were much larger for a Matsuoka-Nakai material than for a von Mises one.

The numerically predicted components of velocity $v_{\phi}^{h}$ and $v_{r}^{h}$ depend upon the choice of model parameters and constitutive relation. When using the Matsuoka-Nakai relation, $v_{\phi}^{h}$ varies strongly as a function of wall friction, $\mu_{w}$, ranging from the same order as the radial flow to three or more orders of magnitude smaller. Similar behavior is seen when the von Mises granular criterion is used, but the effect is smaller by orders of magnitude [16]. 


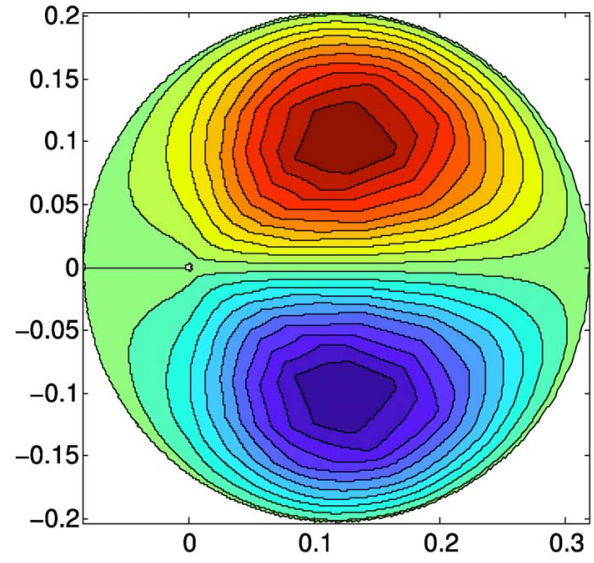

FIG. 1. (Color online) Numerical simulations of the velocity stream functions for tilted hoppers predict the rise of two secondary circulation cells, rotating in opposite directions. Darker tones represent greater velocity. In the top half of the figure the circulation is clockwise, while in the lower half the circulation is counterclockwise. The strength and shape of the cells depend on the angle of tilt, angle of internal friction, and coefficient of wall friction. The azimuthal angle $\phi$ is taken to be zero on the line of symmetry between the two cells.

The numerical model assumes mass flow, where all the grains are moving and there are no stagnant regions within the hopper. For this reason, simulations were not conducted above $\mu_{w}=\tan \left(\theta_{s}\right)=0.5$, where the angle of friction of the simulated material was $\theta_{s}=30^{\circ}[14]$.

\section{METHODOLOGY}

Our experimental test hopper is an approximately conical, regular polyhedron composed of 14 brass wedges $\left(\mu_{w}\right.$ $=0.34$ when bare $)$ and two clear Plexiglas wedges $(\mu$ $=0.51$ ). The hopper, depicted in Fig. 2 , is $\sim 45 \mathrm{~cm}$ wide at the top and the walls are angled at $\theta_{w}=24^{\circ}$. Flow is slowed by a nozzle with a $0.6 \mathrm{~cm}$ diameter opening to $\sim 6 \mathrm{~g} / \mathrm{s}$ and the nozzle is screened from the hopper by a $3 \mathrm{~cm}$ diameter tube that is sufficiently long $(\sim 20 \mathrm{~cm})$ for the influence of the nozzle to be neglected. Sand runs from the opening at the
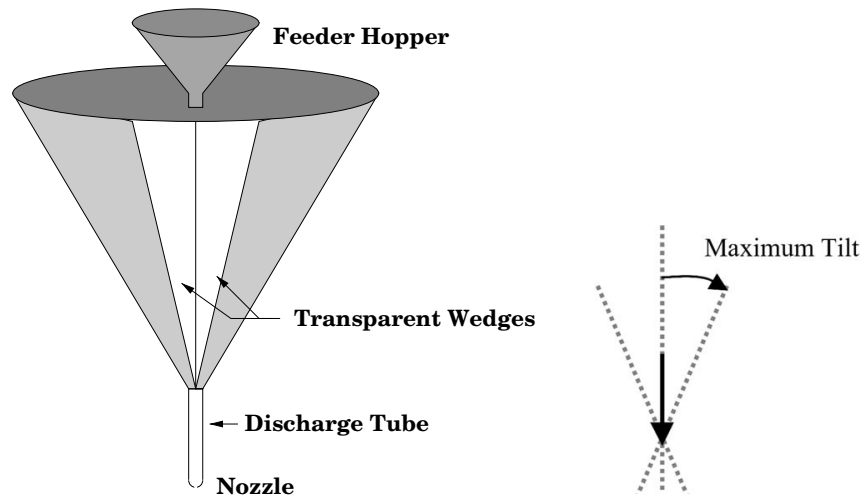

FIG. 2. Schematic of hopper in which we observe flowing sand. To perturb the flow we tilt the hopper up to $23^{\circ}$. bottom of the hopper into the discharge tube, with foam wrapped around the joint to prevent spillage. It takes $\sim 20$ min for the hopper to drain if not refilled. A second, feeder hopper is placed on axis at the top to provide refilling from an approximate point source $\sim 14 \mathrm{~cm}$ within the hopper. The feeder hopper is refilled by hand as needed and keeps the main hopper nearly fixed at roughly one-third full. By feeding from the small hopper we maintain a wellcontrolled preparation of the sand in the primary hopper.

We note that the 16-sided cross section of the hopper involves some perturbation from circular symmetry. However, we estimate that these are 15 to 20 times smaller than the typical perturbation that we achieve by tilting the hopper. In addition, we expect that any small flows induced by these perturbations would not have a substantial swirling component.

In these experiments we use Ottawa sand (ASTM C-190). The sand (coefficient of friction $\mu_{\text {sand }}=0.42$, density $1.64 \pm 0.01 \mathrm{~g} / \mathrm{mL}$, and diameter $420 \leq d \leq 595 \mu \mathrm{m})$ is mixed with a small quantity of tracer sand that has been dyed dark red with instrument ink and repeatedly baked dry.

The entire hopper can be physically tilted, perturbing the geometry with respect to gravity. The feeder hopper tilts with the system, staying on the axis of the main hopper. A digital (CCD) camera, bolted to the side of the hopper, tilts with the hopper and can be aligned with the Plexiglas "windows" in one of two configurations so that images showing the passage of sand may be recorded with a computer. The hopper is tilted so that the windows are on the axis of the tilt, where $v_{\phi}$ is predicted to be maximal. A plumb line is hung within the hopper and imaged to determine the direction of gravity in the frame of the camera for each tilt.

Since the hopper is made of flat wedges shaped into a cone, each wedge has a different orientation relative to the camera. To account for the tilt of the camera relative to the orientation of the window wedges we mount the camera two different ways. In the first, we align the camera so that the tilts of the two windows are equal but opposite in orientation relative to the camera. In this arrangement, any geometric effect caused by the tilt of one window should be reversed in the other window.

We use the second camera arrangement when we line the walls of the hopper with materials with different coefficients of friction. To minimize the impact of the viewing window when lining the hopper we cut a hole no bigger than the size of the field of view of the camera into the liner. In this case we align the camera flush with just one window of the hopper.

The coefficient of friction, $\mu_{w}$, of a particular liner material is determined by using a weight and pulley system to drag a tray covered with the material across a bed of Ottawa sand. We capture the motion of the tray using a high-speed digital camera and analyze the images to determine the acceleration of the tray. For each run there is a period where the forces on the sled are roughly constant and we perform a linear fit to the velocity in this region to determine acceleration. By varying the load applied to the tray as well as the weight acting through the pulley we can account for systematic frictional forces and deduce the sliding coefficient of friction for sand on the liner material. 

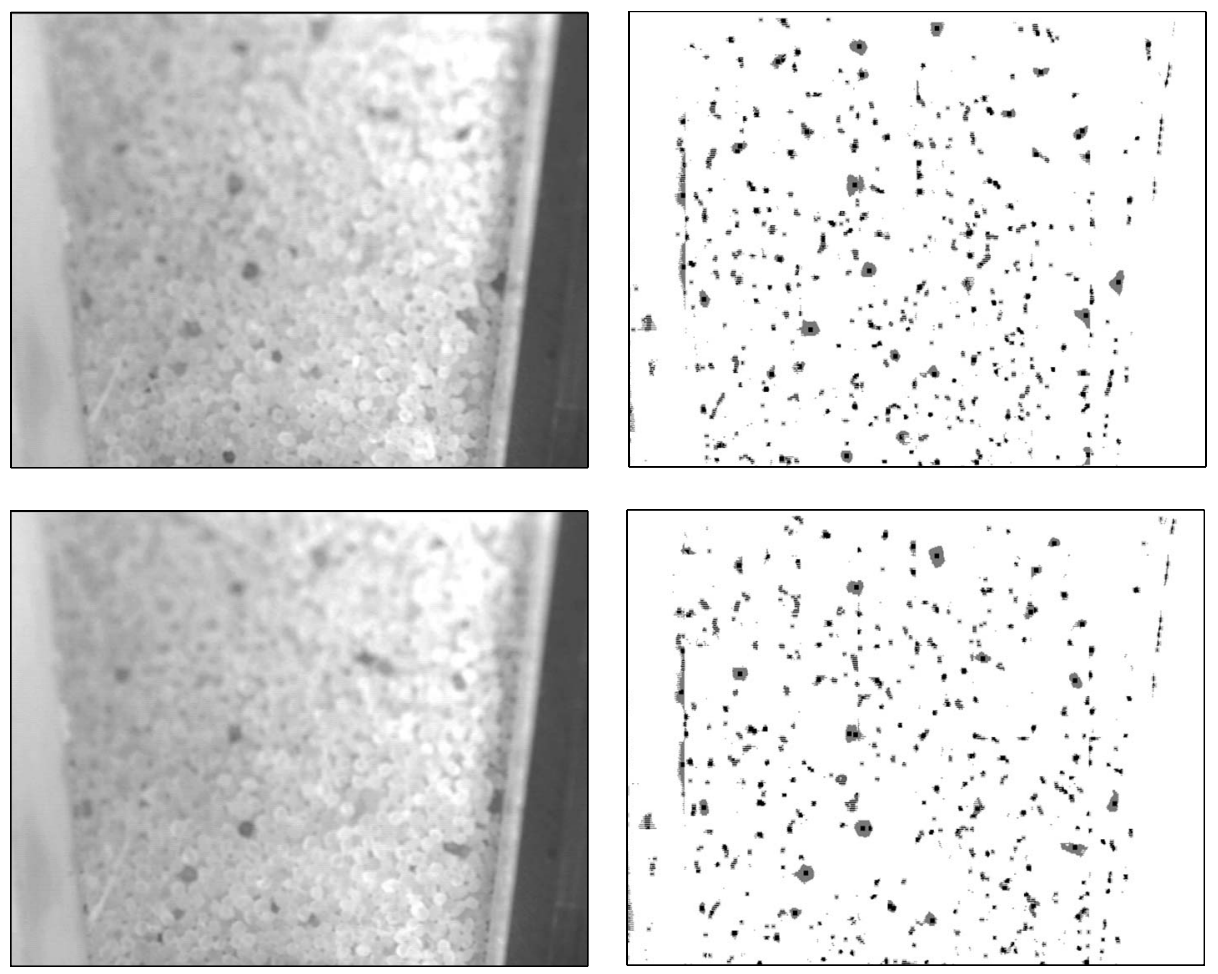

FIG. 3. Velocities are determined from identifying tracer particles and regressing over 21 consecutive frames to assign a velocity to the 11th frame. Shown here are the actual frames (left) and thresholded tracer bitmaps (right) for a given frame (top) and the 20th frame later (bottom), corresponding to $40 \mathrm{~s}$ of experimental observation. The dark dots indicate centers assigned to large tracer particles.
Data sets for determining the velocity field typically consist of 1000 frames taken once every $2 \mathrm{~s}$-covering roughly 33 min of flow. For a given combination of tilt and wall material, from two to six data sets are used to determine each plotted point for $v$.

As illustrated by Fig. 3, particle tracking is performed by thresholding each frame into a binary intensity image where "ones" identify pixels with intensity that is a tunable number of standard deviations away from the local mean intensity in the region of the pixel. The thresholding is done by region to account for any nonuniform lighting. Clusters of "ones" larger than a tunable size are then identified as the dark, tracer particles amidst the lighter sand, although occasionally bright or reflective sand grains are also resolved and tracked. Typically a dozen or more grains are tracked in a frame.

Since the grains are slightly irregular, the cluster identifying a given tracer grain changes as the particle rotates along the window surface. Typical tracer particles are roughly 20 $\times 20$ pixels in size, though smaller dark or bright spots are tracked and used if possible. Each cluster is assigned a "center of mass" determined from the spatial distribution of its pixels, and then is identified with the nearest center of mass in the previous frame to construct a track of particle position in consecutive frames. If the nearest center of mass is outside a tunable maximum radius, the identification is rejected and a new particle track begins. Once a track is 21 frames long, a velocity is calculated using a least-squares fit and the interpolated velocity is assigned to the location of the tracer in the 11th frame, as in Fig. 4, where out of the dozens of possible tracers only three are being used. If the velocity is zero, indicating an edge of the window is being tracked, it is ignored. Particle tracks begin and end as tracer particles move into and out of the field of view of the camera due to the overall flow and motion toward and away from the window - the mean track length is roughly 26 frames. In this manner we generate a time-integrated velocity field by binning velocities into regions by tracer location over the length of the run.

Once a velocity field is generated for a particular combination of tilt angle and wall friction, as in Fig. 5, we can calculate the ratio $v_{\phi} / v_{r}$. If we have recorded using two, off-setting windows, we calculate the ratio separately for the two windows. We first linearly fit both components of velocity horizontally (fixed $r$ ) across the velocity field to the distance from the middle of the image to compensate for the slight tilt of the windows away from the camera. We interpolate using this fit to find the velocity components nearest the middle of the field of view of the camera from both windows, corresponding to $\phi=\pi / 2$ ( $\phi=0$ is in the plane of the tilt). If we are using a single window, we perform a quadratic fit across the velocity field to account for $\phi$ varia-

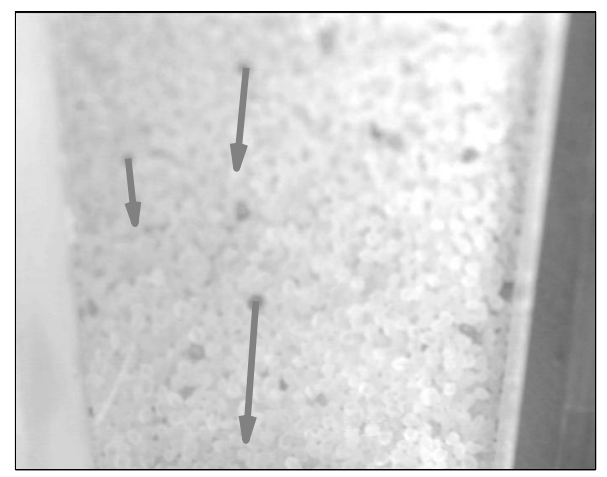

FIG. 4. Velocities assigned to the 11th frame in the sequence depicted in Fig. 3 indicate that in that frame only three tracer particles had sufficiently long unambiguous tracks to be used. 


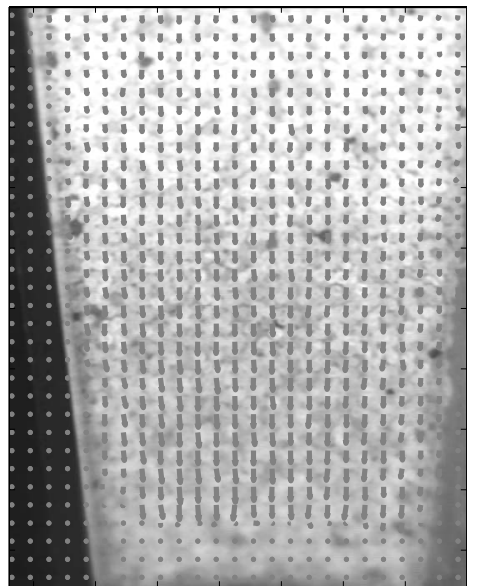

FIG. 5. Time-averaged velocity field is generated by spatially binning the velocities for every frame.

tion (a higher order effect than in the two-window case). We again interpolate the velocity components at $\phi=\pi / 2$.

We find both the azimuthal and radial components of the velocity to vary radially as $1 / r^{2}$, in agreement with mass conservation and the Jenike solutions. Given that both components vary as $1 / r^{2}$, the ratio of the two velocity components, $v_{\phi} / v_{r}$, should be independent of $r$. Linear fits to $1 / r^{2}$ of the two velocity components are made and for each radial bin of the vector field the ratio of the $v_{\phi}$ fit to the $v_{r}$ fit is calculated and the mean is recorded as the observed ratio. Error bars are established using the standard deviation of these ratios.

\section{RESULTS}

We find evidence for a nonradial component of the velocity field as a function of hopper tilt and wall friction. We also find that small perturbations of geometry, such as slight misalignment of the wedges forming our test hopper, causes a small amount of apparent secondary circulation. With careful alignment and balancing of our untilted experimental hopper we were able to reduce, but not completely eliminate, this apparent nonradial flow even for the untilted case. In order to compensate for the observed small nonradial component of the flow, we rotate the experimental images to a frame in which the untilted hopper flow is perfectly radial.

As the measurements from our test hopper shown in Fig. 6 indicate, in a bare brass hopper $(\mu=0.34)$ secondary circulation does in fact follow linearly with tilt angle for small perturbations $(\epsilon<0.15)$. At larger angles, the dependence appears to depart from linear, which might be expected, since higher-order terms should eventually become important. Note that the ratio of azimuthal to radial velocity, $v_{\phi}^{h} / v_{r}^{h}$, follows systematically for tilts in both directions, indicating that our observations are not an artifact.

Numerically it is possible to predict $v_{\phi}^{h} / v_{r}^{h}$ as a function of $\mu_{\text {sand }}, \theta_{\text {wall }}$, and $\mu_{\text {wall }}$, although there are many domains where the predicted magnitude of secondary circulation is too small to be observed. For our system, wall friction is the most readily tunable parameter and we observed the flow for

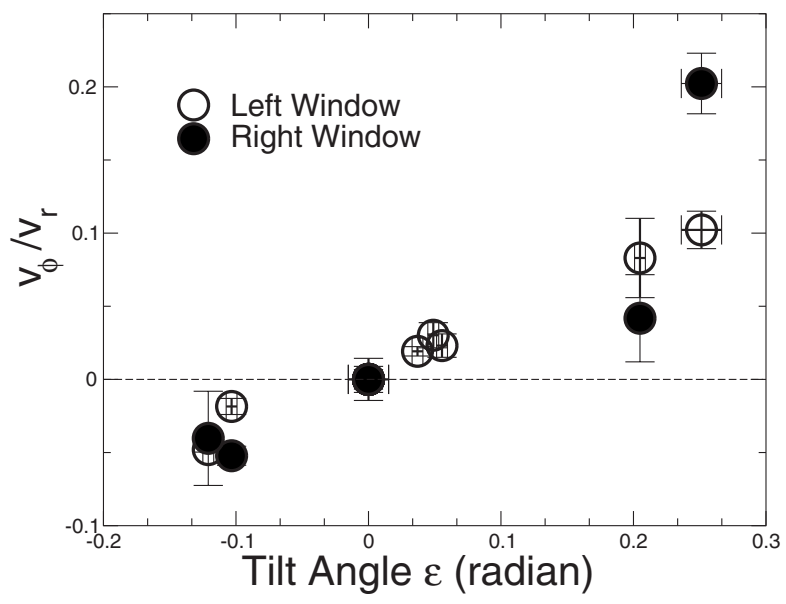

FIG. 6. Ratio of azimuthal to radial velocity for the rightand left-hand $(\bigcirc)$ windows for a bare $\left(\mu_{w}=0.34\right)$ hopper.

three additional wall frictions, as indicated by Fig. 7 .

By choosing the appropriate material, we were able to examine circulation in regimes where azimuthal flow would be suppressed. One such domain is that of low wall friction values (such as Teflon- $\mu=0.05$ ). In the linear region $(\epsilon$ $<0.1)$ there is no detectable secondary circulation, within experimental resolution, when the hopper is lined with Teflon. As the tilt increases however, a strong azimuthal component is observed in the opposite direction of the flow in the bare hopper. The tilt angle for the onset of flow showed some variation, possibly indicating hysteretic effects or some other influence beyond the controls of the experiment.

As wall friction is increased beyond $\mu_{\text {critical }}$, soil mechanics models of granular flow predict a transition from mass flow-where all the material in the hopper is moving-to funnel flow where there are nonmoving, stagnant regions at the wall. However, when we line the hopper with Emery paper, which has a higher coefficient of friction $\mu=0.75$ than the interparticle friction $\mu_{\text {crit }}=\tan \theta_{\text {sand }}$, we continue to ob-

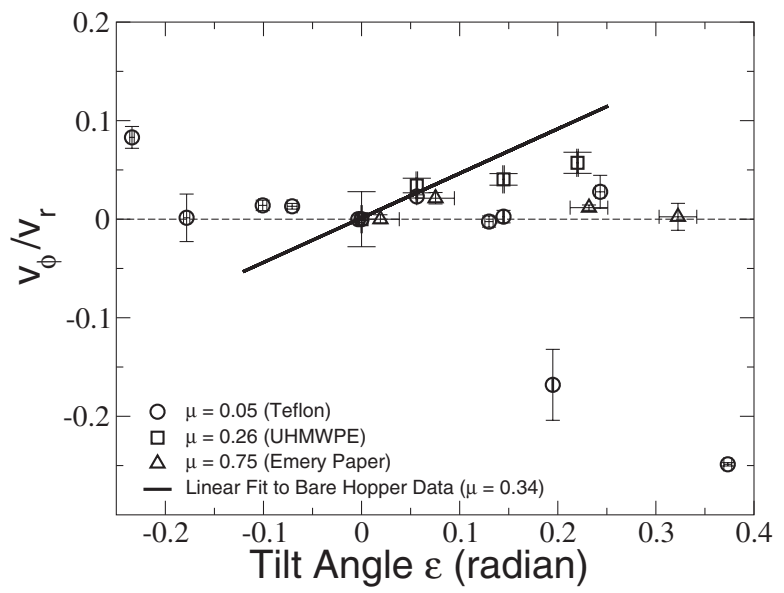

FIG. 7. Comparison of the ratio of azimuthal to radial velocity for Teflon $\left(\mu_{w}=0.05-\bigcirc\right)$, UHMWPE $\left(\mu_{w}=0.26-\square\right)$, and Emery paper $\left(\mu_{w}=0.75-\triangle\right)$ to a linear regression of the bare $\left(\mu_{w}=0.34\right)$ hopper observations (solid line). 


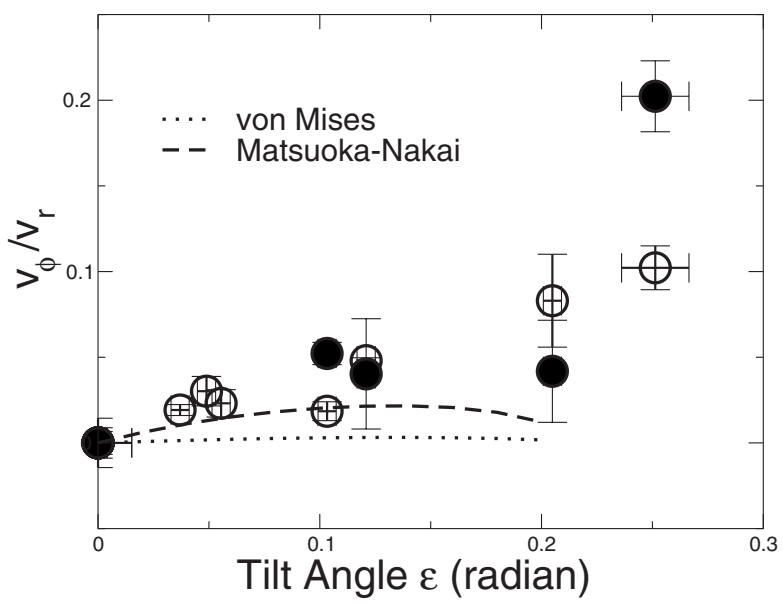

FIG. 8. Ratio of azimuthal to radial velocity for a bare hopper as a function of hopper tilt angle $\epsilon$ as imaged through the right $(\mathbf{)}$ and left $(\bigcirc)$ windows of the test hopper, rotated to the frame where the untilted flow is entirely radial and mapped so that all tilt angles are positive. Lines indicate numerical predictions for von Mises (dotted) and Matsuoka-Nakai (dashed) plasticity models.

serve some radial flow at the window, although there is no azimuthal component within measurement error.

Although extreme values of wall friction induce large changes in flow, we find that the flow is relatively robust to small variations in wall friction. Changing the wall friction slightly from the bare hopper (from $\mu_{w}=0.34$ to 0.26 ), by lining with low-friction ultrahigh molecular weight polyethylene (UHMWPE), does not qualitatively change the secondary circulation.

\section{COMPARISON WITH SOIL MECHANICS PREDICTIONS}

The dashed and dotted lines in Fig. 8 show azimuthal to radial velocities ratios as calculated for varying tilts in a bare hopper using a spectral method [16]. We examine the ratio at the point in the hopper where we observed experimentally. As the tilt increases, we predict the onset of stronger and stronger circulation. In this respect, the predicted behavior of the simulation and experiment are similar for small tilt angles.

As the tilt increases further, we see in the simulations that the circulation cells move relative to the point on the hopper wall where we have observed the flow experimentally. This movement of the circulation cells puts the observation area at a point where the rotational flow is much smaller and approaches zero. This decrease in the flow is not observed experimentally.

We find that the values predicted using the MatsuokaNakai constitutive relation match our observations reasonably well for small angles, $|\epsilon|<0.1$, while the granular von Mises relation predicts much too little circulation. This suggests that the Matsuoka-Nakai criterion may more accurately describe the yielding of dense granular flows in the regime we are studying.

When the hopper wall friction is lowered (e.g., Fig. 7, Teflon lining) the ratio $v_{\phi} / v_{r}$ is numerically predicted to be

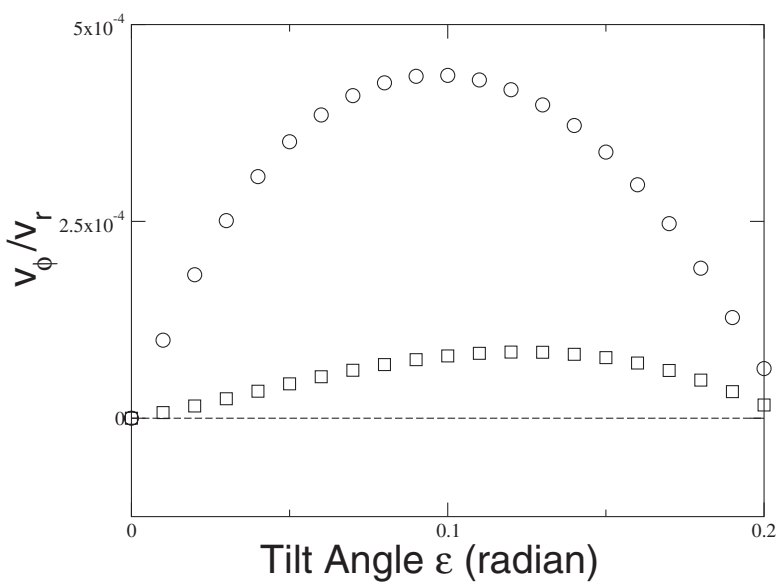

FIG. 9. When wall friction is extremely low, $\mu_{w}=0.05$, the numerically predicted ratio of azimuthal to radial velocity as a function of hopper tilt angle for both the Matsuoka-Nakai $(\bigcirc)$ and von Mises $(\square)$ criteria is four orders of magnitude smaller than the experimentally observed ratio at large tilt angles.

very small for both the Matsuoka-Nakai and von Mises yield criteria, as shown in Fig. 9. Both sets of predictions are beneath the threshold of detection in this regime and we cannot distinguish between them experimentally. We note, however, that we observe no trend for $|\epsilon|<0.2$ in Fig. 7.

\section{VELOCITY FLUCTUATIONS}

Although the numerical approach here describes the mean flow, we know that stress in hoppers is subject to large fluctuations about the mean [12]. Since Levy's flow rule assumes that the velocity in our hopper is directly related to the stress, it is interesting to examine any fluctuations in our velocity distributions [17].

Previous work by Zhu and Yu using numerical discrete element methods has studied the probability distribution of velocities in a flat-bottomed cylindrical hopper as wall friction was varied from $\mu=0.1$ to 0.5 [19]. Although their cylindrical system had notable differences from ours, including the presence of stagnant regions and plug flow, separate distributions for the radial component of flow were calculated, allowing rough comparison. In the DEM simulations it was found that the fluctuations decrease as wall friction is reduced.

In Fig. 10 we show the probability density distribution of radial velocity in an untilted hopper for the four wall frictions we examined. We generate our velocity histograms by scaling several sets of data by the mean radial velocity for that set. Since the mean velocity varied for some experiments, for each value of wall friction we use the largest data set with consistent radial velocities to generate our histograms. Within one data set, the velocity fluctuates sharply with time due to both our tracking technique which can produce frames in which no velocities are assigned and the large variability inherent to dense granular flows $[20,21]$. The power spectrum for the time series does not indicate periodicity. 

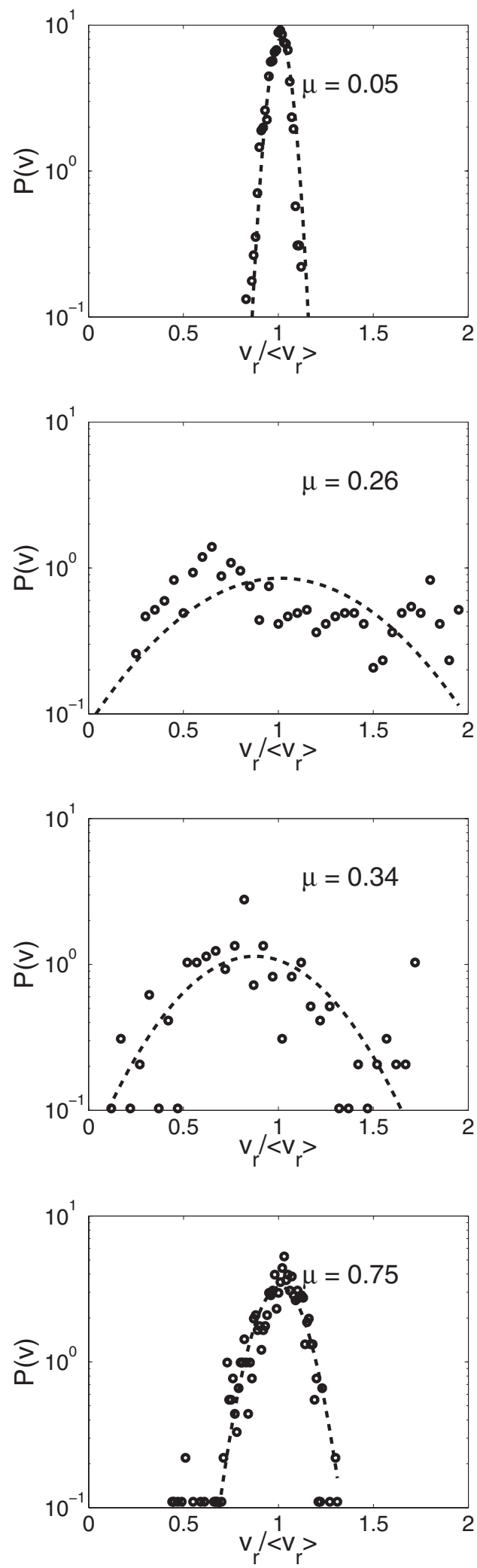

FIG. 10. Width of the distributions of probability density for radial velocity varies nonmonotonically with wall friction, with the extremes having much narrower distributions than more moderate friction values. The distributions are roughly fit by Gaussians (dashed lines), with moderate values of friction having positive skewness and extreme values having negative skewness.
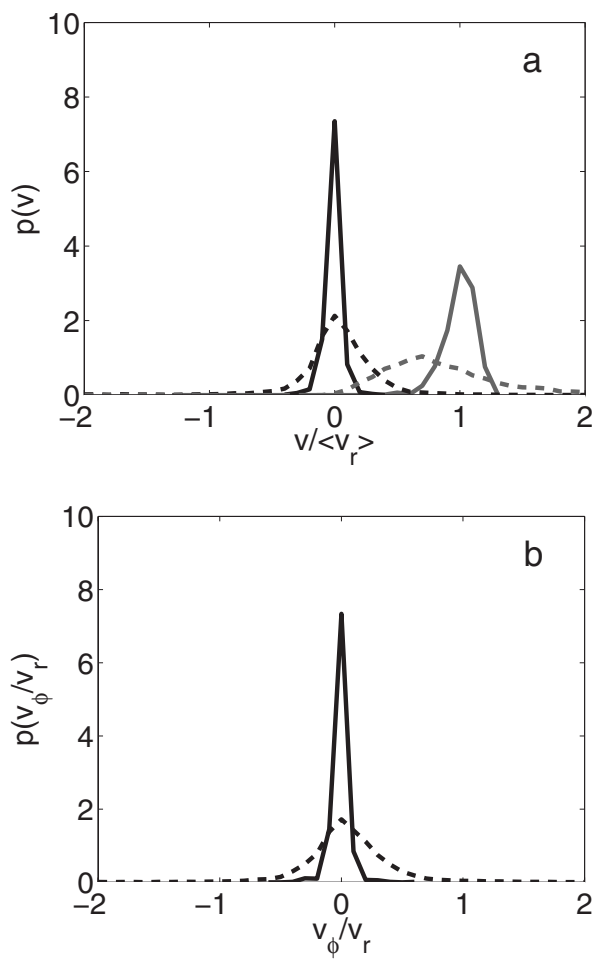

FIG. 11. Probability density distributions of (a) the azimuthal (left) and radial (right) components of the velocity and (b) $v_{\phi} / v_{r}$ for an untilted hopper (solid line) and a hopper at tilt $\epsilon=0.14$ (dashed line). These results are for an Emery paper-lined hopper, although they are representative of other wall frictions.

As friction is increased from moderate values we observe a decrease in the overall velocity fluctuations in agreement with the numerical predictions. The coefficient of friction for Teflon is below the range studied previously, and in that case we observe that the fluctuations are suppressed relative to moderate wall frictions, indicating a nonmonotonic dependence of velocity fluctuations on wall friction. All our observed distributions have skew, positive for moderate friction and negative for extreme frictions, but because we have studied velocities interpolated from many frames we do not see the wide distributions of Moka and Nott [22].

Finally, we examined the distribution of velocities as a function of tilt angle. For all wall linings, independent of the presence of secondary circulation, we observed an increase in fluctuations around the mean values as the tilt angle was increased. Figure 11 shows the probability distributions for the radial and azimuthal components of the velocity as well as the ratio of those two components for untilted and highly tilted cases. For both tilted and untilted hoppers the means are similar, but the distribution grows much wider with tilt angle. Interestingly, although the distributions are wider for the highly tilted hopper, the distribution of $v_{\phi} / v_{r}$ is of roughly the same width as that of $v_{\phi}$, indicating that the fluctuations of the two components are correlated. This is associated with the fact that individual grains follow roughly fixed trajectories even if they are different from the mean flow. These persistent trajectories may indicate long-term correlation of particle contacts that might be expected for 
clusters of grains moving together, such as the "granular eddies" of Ertaş and Halsey [23].

\section{CONCLUSION}

We have observed that real three-dimensional flows of granular matter are more complex than the idealized case of perfectly radial Jenike-like flow. In response to small perturbations (in the form of tilts), nonradial velocities arise leading to potentially large shifts in behavior. For small tilts we have found that the magnitude of circulation numerically predicted using the Matsuoka-Nakai constitutive relation is closer to observations than numeric predictions made with the more traditional, generalized von Mises condition. At larger tilts we observe that the nonradial flow becomes even more pronounced, exceeding numerical predictions for all constitutive relations.

We have examined the influence of wall friction and discovered that extreme values, low or high, act to suppress secondary circulation. In the case of large perturbations and low friction, we see an abrupt and unpredicted onset of secondary circulation in the opposite direction of the moderate friction case.

Finally, we have observed that the fluctuations of individual grain velocities grow as tilt is increased. We find this to be true independent of lining materials. We also note that a single grain may follow a trajectory that is quite different from the mean flow for long periods of time, which produces large, correlated fluctuations. Such particle-scale fluctuations are not captured by continuum models, but do not seem to affect the mean behavior.

\section{ACKNOWLEDGMENTS}

J.F.W. and R.P.B. acknowledge funding from National Science Foundation Grants No. DMR-0137119, No. DMR0555431, and No. DMS-0204677 and NASA Grant No. NNC04GB08G. P.A.G. acknowledges funding from NSF Grants No. DMS-0244488 and No. DMS-0410561.
[1] R. Nedderman, Statics and Kinematics of Granular Materials (Cambridge University Press, Cambridge, UK, 1992).

[2] H. Matsuoka and T. Nakai, Soils Found. 25, 123 (1985).

[3] H. Matsuoka, T. Hoshikawa, and K. Ueno, in Powders and Grains, edited by J. Biarez and R. Gourvés (Balkema, Netherlands, 1989), pp. 339-346.

[4] D. G. Schaeffer, Int. J. Numer. Analyt. Meth. Geomech. 14, 253 (1990).

[5] A. W. Jenike, Tech. Rep. 108, University of Utah, 1961.

[6] J. Choi, A. Kudrolli, R. R. Rosales, and M. Z. Bazant, Phys. Rev. Lett. 92, 174301 (2004).

[7] S. Horlück and P. Dimon, Phys. Rev. E 63, 031301 (2001).

[8] A. Medina, J. A. Córdova, E. Luna, and C. Treviño, Phys. Lett. A 250, 111 (1998).

[9] C. S. Chou, J. Y. Hsu, and Y. D. Lau, Physica A 308, 46 (2002).

[10] J. R. Ducker, M. E. Ducker, and R. M. Nedderman, Powder Technol. 42, 3 (1985).

[11] S. B. M. Moreea and R. M. Nedderman, Chem. Eng. Sci. 51,
3931 (1996).

[12] G. W. Baxter, R. Leone, and R. P. Behringer, Europhys. Lett. 21, 569 (1993).

[13] G. H. Ristow, Ph.D. thesis, Philipps University-Marburg, 1998.

[14] P. A. Gremaud, J. V. Matthews, and D. G. Schaeffer, SIAM J. Appl. Math. 64, 583 (2003).

[15] P. A. Gremaud, J. V. Matthews, and M. O’Malley, J. Comput. Phys. 200, 639 (2004).

[16] P. A. Gremaud, J. V. Matthews, and D. G. Schaeffer, J. Comput. Phys. 219, 443 (2006).

[17] A. W. Jenike, Powder Technol. 50, 229 (1987).

[18] D. Howell, Ph.D. thesis, Duke University, 1999.

[19] H. P. Zhu and A. B. Yu, J. Phys. D 37, 1497 (2004).

[20] N. Menon and D. J. Durian, Science 275, 1920 (1997).

[21] E. Gardel, E. Keene, S. Dragulin, N. Easwar, and N. Menon, e-print arXiv:cond-mat/0601022.

[22] S. Moka and P. R. Nott, Phys. Rev. Lett. 95, 068003 (2005).

[23] D. Ertaş and T. C. Halsey, Europhys. Lett. 60, 931 (2002). 